Polymer Journal, Vol. 39, No. 11, pp. 1143-1149 (2007)

(C) 2007 The Society of Polymer Science, Japan

\title{
Electrical Properties of Non-Crosslinked Polyethylene/Syndiotactic Polystyrene Composites Filled with Carbon Black
}

\author{
Keun-Byoung Yoon, Ga Hyoung LeE, Woo Young CHOI, and Dong-ho LEE ${ }^{\dagger}$ \\ Department of Polymer Science, Engineering College, Kyungpook National University, Daegu 702-701, Korea
}

(Received May 18, 2007; Accepted July 27, 2007; Published September 14, 2007)

\begin{abstract}
Carbon black (CB)-filled high density polyethylene (HDPE)/syndiotactic polystyrene (sPS) composites were prepared by the conventional melt-mixing procedure. The effect of the HDPE/sPS weight ratio, CB content and processing speed on the thermoelectric behavior such as positive temperature coefficient (PTC) and negative temperature coefficient (NTC) was investigated in detail. The non-crosslinked HDPE/sPS composites containing 10-phr $\mathrm{CB}$, a typical PTC behavior was observed when the temperature increased toward the melting point of HDPE. At high processing speed, the composites exhibited high room temperature resistivity and high PTC intensity. In a high sPS content composite, the dispersion of sPS particles are homogeneous and the distance of sPS particles are very close. The CB-coated sPS particles could be formed a continuous conductive network; therefore the PTC effect became weaker and weaker in these systems. The elimination of the NTC effect in CB-filled composites can be achieved by using a very high melting semicrystalline polymer as one of its components. [doi:10.1295/polymj.PJ2007046]

KEY WORDS Non-crosslinked / Polyethylene / Syndiotactic Polystyrene / Carbon Black / Positive Temperature Coefficient (PTC) / Negative Temperature Coefficient (NTC) /
\end{abstract}

An interesting phenomenon exhibited by the some conductive polymer is a positive temperature coefficient (PTC) effect. The main feature of PTC materials is that heating causes the conductive system to show a sharp resistivity increase near the melting region of a semicrystalline polymer matrix. ${ }^{1,2}$

A well-known technique that brings conducting properties into an intrinsically insulating polymer incorporates it with conducting fillers such as carbon black, ${ }^{3}$ metal powder and fibers ${ }^{4}$ or graphite fibers. ${ }^{5}$

Although metal powders are intrinsically more conductive than carbon black, metal has a tendency to oxidation to form an insulating layer on its surface and is not used as frequently as carbon back (CB). One of the most common additives used in conducting polymers is $\mathrm{CB}$, which initially forms isolated structures within the matrix; then isolated clusters form, and finally through-going paths arise.

The critical amount of CB necessary to form a conductive network is referred to as the percolation threshold. It is desirable for the conducting filler content to be as low as possible to achieve good processability, good mechanical properties and low cost.

It is well known that when the temperature is above the melting point of semicrystalline polymers, noncrosslinked CB-filled semicrystalline polymer composites exhibit a very sharp decrease in resistivity. This phenomenon is referred to as the negative temperature coefficient (NTC) effect. ${ }^{6,7}$

In general, non-crosslinked CB-filled semicrystalline polymer composites cannot be used as thermistors in over-temperature and over-current protections due to their NTC effects and poor reproducibility in thermal recycling. To overcome these disadvantages, researchers have proposed and developed many methods to eliminate the NTC effect of CB-filled semicrystalline polymer composites. Among these methods, the approach used to crosslink the semicrystalline polymer matrix by a crosslinking agent, such as a peroxide $^{8}$ or silane compound. ${ }^{9}$ In addition to the use of a crosslinking agent, Gamma and electron beam radiations have been used to crosslinked CB-filled semicrystalline polymer composites. ${ }^{10-12}$

Using $\mathrm{CB}$ as an additive to achieve electrical conductivity usually requires a concentration so high that it will increase the melt viscosity and decrease the mechanical properties of the polymers. One of the recent trends is to use multiphase polymer blends to reduce the amount of conductive fillers in composites. ${ }^{13-15}$

The phase separation for polymer blends, and selective distribution of $\mathrm{CB}$ into one phase, can produce conducting material at lower filler content. This method can also provide better mixture processability and avoid poor mechanical properties caused by high filler loading.

Recently, CB-filled polypropylene (PP) composites were prepared by addition of ultra high molecular weight polyethylene (UHMWPE) as a third filler. This non-crosslink CB-filled PP/UHMWPE composites did not show any NTC effect, the elimination of the NTC effect is attributed to the extremely high viscosity of UHMWPE. ${ }^{16}$ Although the elimination of the

${ }^{\dagger}$ To whom correspondence should be addressed (Tel: +82-53-950-5625, Fax: +82-53-950-6623, E-mail: leedh@knu.ac.kr). 
NTC effect is not well understood, it is agreed that the NTC effect is related to the significant reduction of the mobility of the $\mathrm{CB}$ embedded in a melt. ${ }^{17}$

In this study, non-crosslinked CB-filled high density polyethylene (HDPE)/syndiotactic polystyrene (sPS) composites were prepared by the conventional melt-mixing process. The blend is an immiscible system comprising a low melting point semicrystalline polymer (HDPE) and high melting point semicrystalline polymer (sPS). The effects of the HDPE/sPS weight ratio and CB content on the PTC behaviors of the composite were investigated. The effect of sPS on the electrical conductivity, morphology and thermal properties of blends were also investigated.

\section{EXPERIMENTAL}

The principle polymers used in this study were HDPE (Seetec, PH143, $\mathrm{T}_{\mathrm{m}}=128^{\circ} \mathrm{C}$ ) and sPS (Dow Chemical, XU 72104.04L, $\left.\mathrm{T}_{\mathrm{m}}=265^{\circ} \mathrm{C}\right)$. As the conductive filler, electrically conductive CB (Cabot, Black Pearl 2000) was used.

All blend ratios described in the text relate to weight ratios. Blends were prepared by a standard procedure of melt-mixing the dry-blended $\mathrm{CB}$ and polymer components in a Brabender plasticorder (PLE 331 ) equipped with a $50 \mathrm{~cm}^{3}$ cell at $300^{\circ} \mathrm{C}$. The resulting blends were subsequently compression-molded into $100 \mu \mathrm{m}$ thick sheets using a hot press at $300^{\circ} \mathrm{C}$.

The sample size for the measurements of the resistivity was $10 \mathrm{~mm}(\mathrm{H}) \times 10 \mathrm{~mm}(\mathrm{~W}) \times 100 \mu \mathrm{m}(\mathrm{T})$. The resistivity along the sample length as function of temperature was measured using a multimeter (HP 34401A) and a programmable oven. The samples were heated at a rate of about $1{ }^{\circ} \mathrm{C} / \mathrm{min}$. Prior to the electrical measurements, silver paste was coated on the samples as the electrode material reduce to contact resistance. ${ }^{18}$

The melting behavior of the composites was determined using a differential scanning calorimeter (DSC, TA 10) at a heating rate of $20^{\circ} \mathrm{C} / \mathrm{min}$ under an inert nitrogen atmosphere.

The morphology of the composites was investigated by using scanning electron microscope (SEM, Hitachi) after fracture of the samples in liquid nitrogen.

\section{RESULTS AND DISCUSSION}

The effect of CB content on the PTC behavior of non-crosslinked HDPE/sPS composites has been studied extensively. Generally, low CB content composites show a higher room temperature resistivity and a higher PTC intensity, however higher CB content composites exhibit a lower room temperature re-

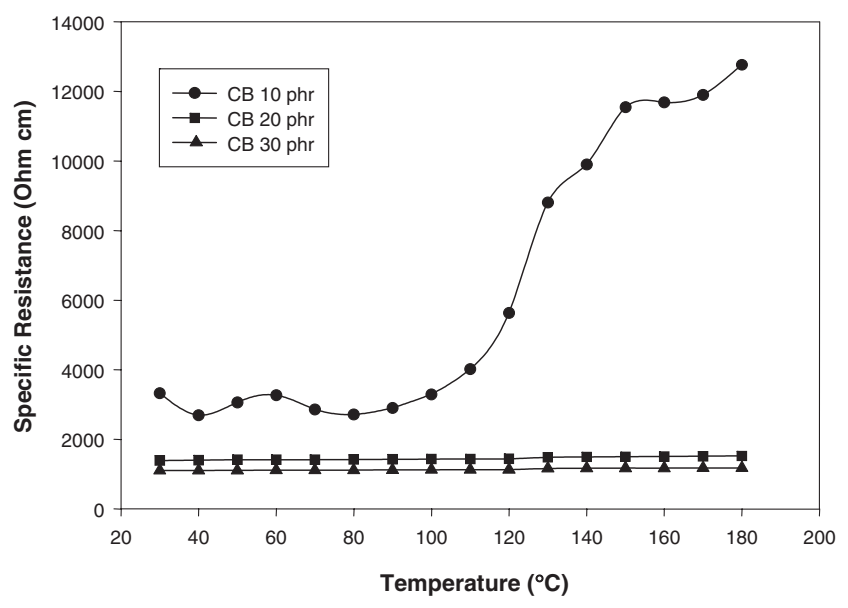

Figure 1. Effect of carbon black content on specific resistance of HDPE/sPS (50/50) composites which were prepared at $35 \mathrm{rpm}$.

sistivity and a lower PTC intensity. Figure 1 displayed the resistivity of non-crosslinked HDPE/sPS (50/50) composites with various $\mathrm{CB}$ content as a function of temperature.

The non-crosslinked HDPE/sPS (50/50) composites containing 10-phr (parts per hundred parts by weight) CB, a typical PTC behavior was observed when the temperature increased toward the melting point of HDPE. At the CB content of 10-phr, the composite exhibited a high room temperature resistivity and a high PTC intensity. As depicted by Figure 1, upon heating, sample went two jumps in resistivity. The HDPE/sPS (50/50) composite containing 10phr CB showed a sharp jump in resistivity as the sample was heated through the HDPE melting region at about $120^{\circ} \mathrm{C}$. Surprisingly, although the sPS peak melting temperature occurs at about $270^{\circ} \mathrm{C}$, the second PTC effect occurs at about $170^{\circ} \mathrm{C}$. The first PTC intensity controlled by the thermal expansion of HDPE, whereas the second PTC intensity controlled by the commencing melting of the sPS. The second resistivity jump's maximum was not attained and therefore the NTC effect could not be observed, due to the sample prior collapse. ${ }^{19}$ The double resistivity jump phenomenon (double PTC effect) was previously observed by Feng and Chan for PP/ UHMWPE/CB system. ${ }^{18}$

When the temperature increased the vicinity to the melting point of HDPE, a large thermal expansion of the polymer occurs as a result of the melting of the crystallites, producing a high PTC intensity. This phenomenon is caused by the two factors, the interparticle distance and the number of conductive paths. ${ }^{6,20,21}$

The large thermal expansion of the polymer matrix can significantly increase the interparticle distance and reduce the number of conductive paths. According to the tunneling theory, the tunneling probability of an electron is related to the interparticle distance. ${ }^{22}$ 
Therefore, the resistivity increases dramatically as the interparticle distance increases. The probability of electron tunneling will be very small for the interparticle distance greater than approximately $10 \mathrm{~nm}$. For a composite with a low CB content, a large number of the interparticle distance could be greater than $10 \mathrm{~nm}$ after the thermal expansion, hence, the resistivity of the composite will greatly increase owing to the combined effect of larger interparticle distances and a much smaller number of conductive paths. For a composite with a high $\mathrm{CB}$ content, the $\mathrm{CB}$ particles can form a continuous conductive network or the interparticle distances can be very close. The increase in the interparticle distance is thus likely to be the only factor that causes the increase in the resistivity. As the CB content increases, the PTC effect became weaker and weaker in this system. These results agree well with the data from several reports on CB-filled single semicrystalline polymer composites. ${ }^{7,8,10,16,17}$

As the CB content increases, the composites exhibit a lower room temperature resistivity, while the PTC behavior could not be observed, not followed by any increase with the temperature further increasing, depicting the presence of high-quality conductive paths. ${ }^{19}$ Obviously, the CB content can significantly influence the PTC behavior of the composites.

Figure 1 also depicted the relationship of the PTC intensity and CB contents. The PTC intensity was calculated from logarithm of the ratio of maximal resistivity near $T_{m}$ and room temperature resistivity. ${ }^{23}$ The value of PTC intensity was 0.6 in HDPE/sPS (50/50) composite containing 10-phr CB.

The effect of sPS content on the thermoelectric behavior has been studied at constant CB content (10 phr) and the results are shown in Figure 2.

The HDPE/sPS (50/50) composites had a significantly higher room temperature resistivity, showed a

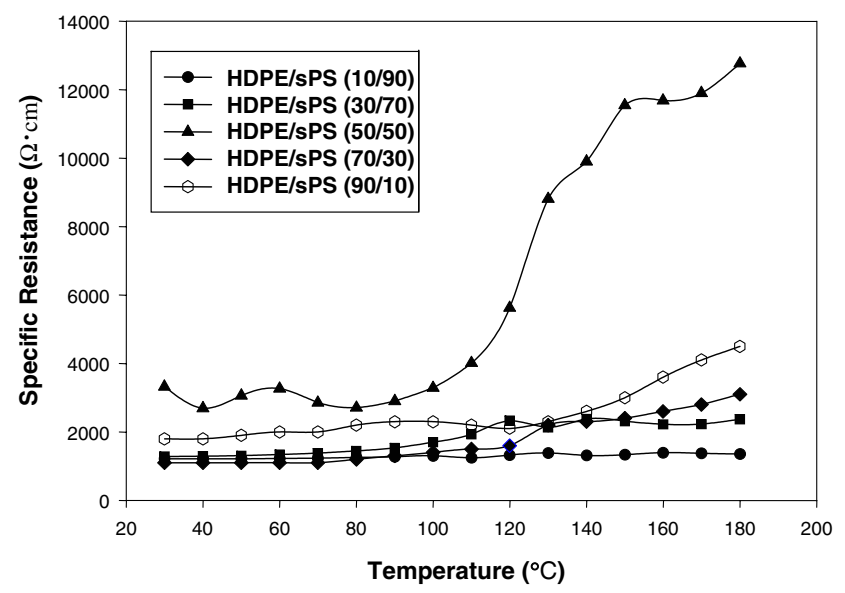

Figure 2. Effect of HDPE/sPS ratios on specific resistance of composites containing $10 \mathrm{phr} \mathrm{CB}$ which were prepared at $35 \mathrm{rpm}$.

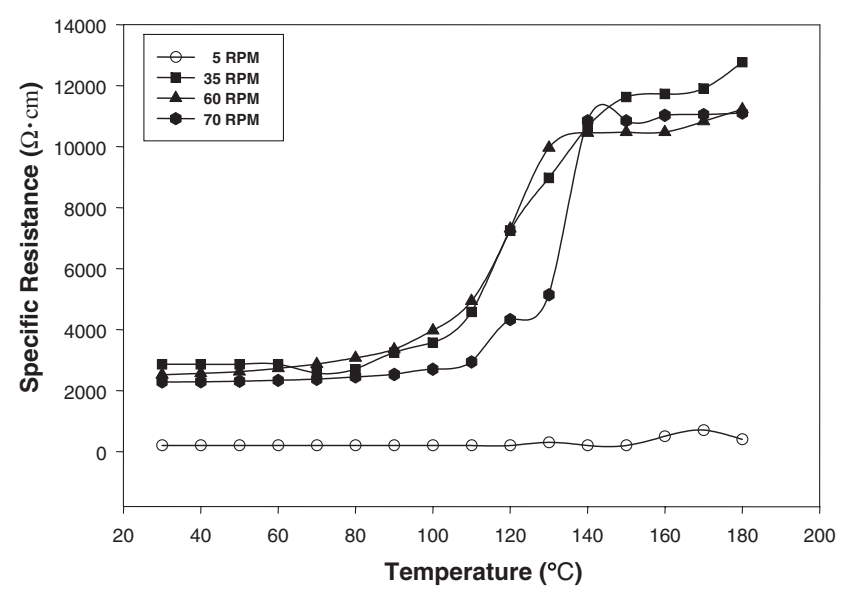

Figure 3. Effect of processing speed on specific resistance of HDPE/sPS (50/50) with 10-phr CB composite.

dramatic increase of resistivity with temperature. This resistivity increase occured at about $120^{\circ} \mathrm{C}$.

The thermoelectric behavior of the HDPE/sPS composites $(90 / 10,70 / 30,30 / 70)$ was similar the room temperature resistivity of the composites is of the same order of magnitude and slightly increased with temperature. However, the composite of HDPE/ sPS (10/90), which had a significantly lower room temperature resistivity with temperature, not followed by any increase with the temperature further increasing, depicting the presence of high quality conductive paths.

The resistivity-processing rate relation was studied for HDPE/sPS (50/50) containing 10-phr CB that was melt-mixed at various speed 5, 35, 60 and 70 rpm. The results are shown in Figure 3.

The processing condition of HDPE/sPS composites can significantly influence the thermoelectric behaviors. At high processing speed, the composites exhibited high room temperature resistivity and high PTC intensity. However, the composite prepared at low processing speed $(5 \mathrm{rpm})$, which had a lower room temperature resistivity, not followed by any increase with the temperature further increasing. As the processing speed increase, the resistivity sharply increased. Because of the homogeneous distribution of sPS domain and the reducing the particle size of sPS domain.

The immiscible blend morphology was found to greatly affect the PTC behavior. ${ }^{24}$ When CB is entirely localized in the matrix, the PTC behavior is essentially the same as in a CB-filled single polymer blend. Another interesting possibility is the localization of CB particles solely at the interface of the immiscible polymer blend. ${ }^{25,26}$

Based on the morphological characteristic of the composites, the PTC behaviors of the composites can be understood. The morphology studied at a dif- 


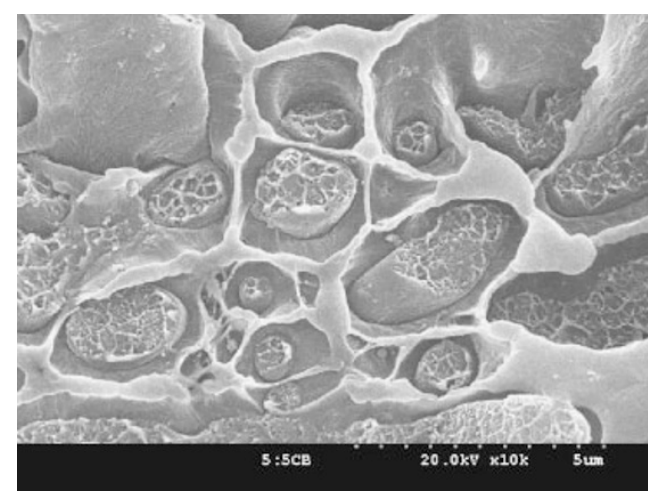

(a)

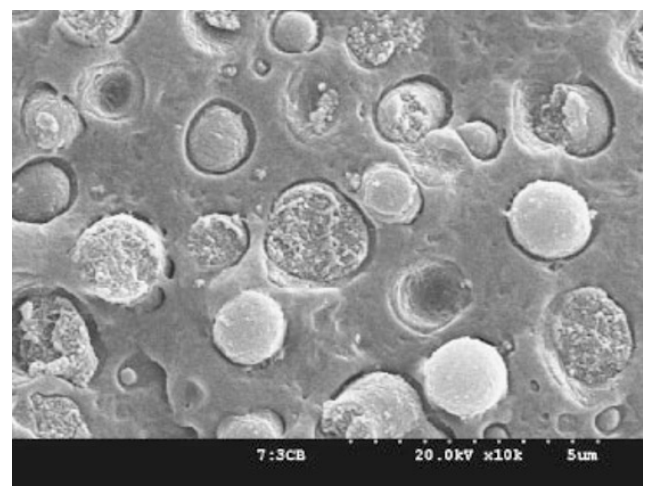

(b)

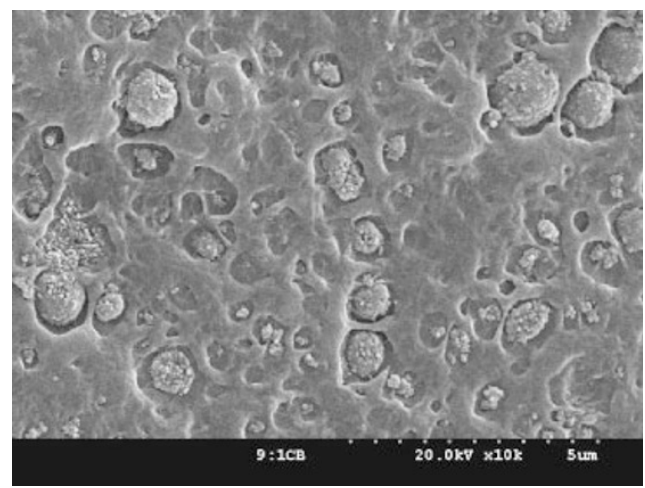

(c)

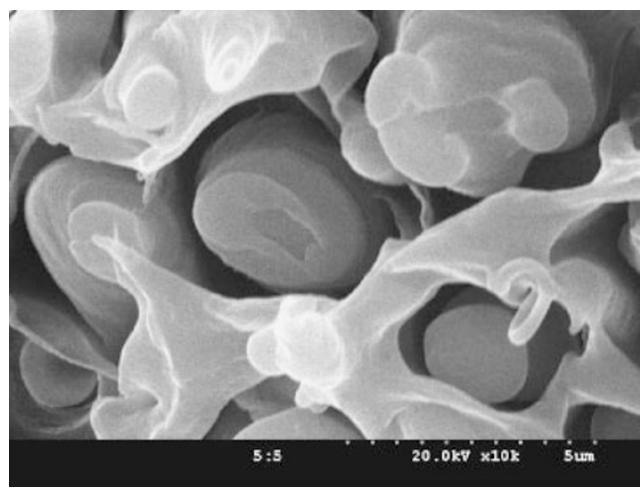

(d)

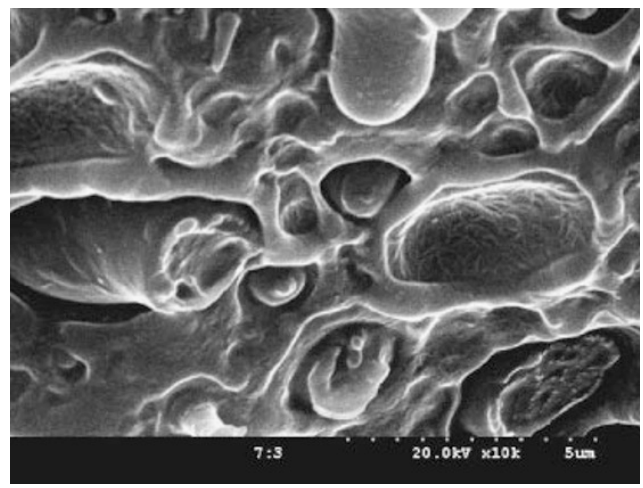

(e)

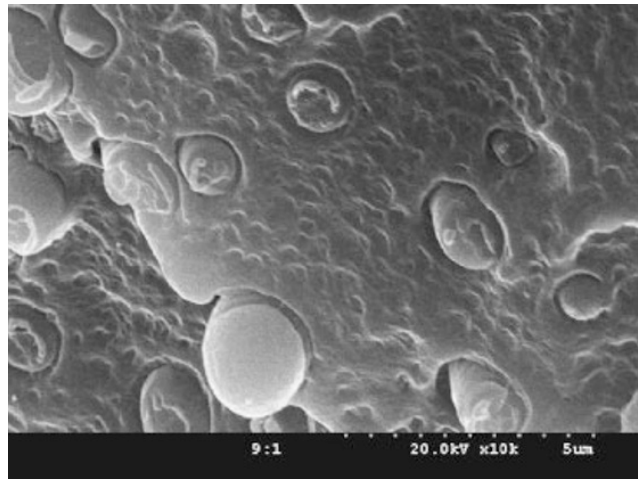

(f)

Figure 4. SEM micrographs of HDPE/sPS composites prepared at $60 \mathrm{rpm}$. (a) HDPE/sPS (50/50) with $10 \mathrm{phr}$ CB, (b) HDPE/sPS (70/ 30) with $10 \mathrm{phr}$ CB, (c) HDPE/sPS (90/10) with $10 \mathrm{phr}$ CB, (d) HDPE/sPS (50/50) without CB, (e) HDPE/sPS (70/30) without CB and (f) HDPE/sPS (90/10) without CB.

ferent ratio of HDPE/sPS composites obtained at $60 \mathrm{rpm}$ and different speed of HDPE/sPS $(90 / 10)$ composites.

The SEM micrographs of $\mathrm{CB}$ filled and unfilled HDPE/sPS composites are shown in Figure 4.

The domains with a relatively rough fracture surface were the HDPE and the domains with a very smooth fracture surface were sPS. The CB filled and unfilled composites consists of spherical sPS droplet inlayed within the HDPE matrix but not adhere to it, as depicted by the gap between the two polymers. In corporation of $\mathrm{CB}$ in small amounts into HDPE/sPS composites, does not alter the morphology of composites compared to the $\mathrm{CB}$ unfilled blends. The adhesion between the two polymers in the $\mathrm{CB}$ presence is better than the one depicted by the neat blend, as evident by the freeze fracture propagating within some of the sPS particles (Figure 4(a) and 4(b)). When the composite cools from its processing temperature at $300^{\circ} \mathrm{C}$, the sPS starts to solidify at around $250^{\circ} \mathrm{C}$ and the HDPE is still in the melt state. The sPS is a hard solid and the contraction of the HDPE phase as a result of crystallization creates gaps between the sPS and the HDPE phase. 


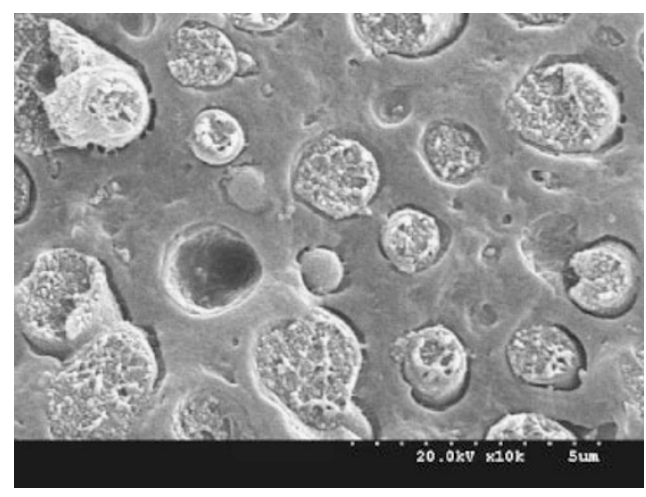

(a)

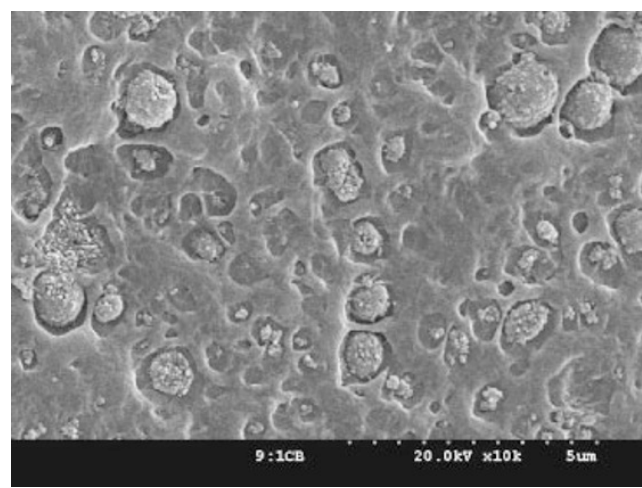

(b)

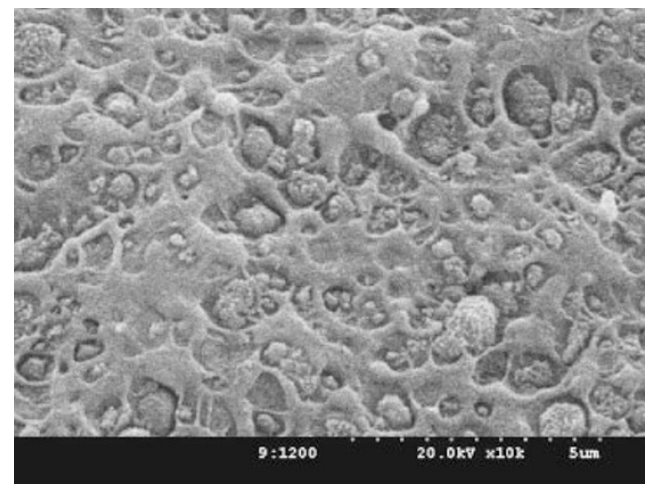

(c)

Figure 5. SEM micrographs of HDPE/sPS (90/10) composites prepared at different mixing speed. (a) $35 \mathrm{rpm}$, (b) $60 \mathrm{rpm}$ and (c) $200 \mathrm{rpm}$.

The spherical sPS domains decreased with increasing the ratio of HDPE/sPS, the diameter of sPS droplet decreased $2.5 \mu \mathrm{m}$ to $0.5 \mu \mathrm{m}$. The domain size of $\mathrm{CB}$ unfilled composites was higher than that of $\mathrm{CB}$ filled composites.

The morphologies of composites prepared at different melt-mixing speed, the SEM micrographs are shown in Figure 5.

The size of sPS particles decreased with increasing processing speed. The sPS particles were too small at high mixing speed, and distribution was homogeneous. The distance of CB coated sPS particle was too

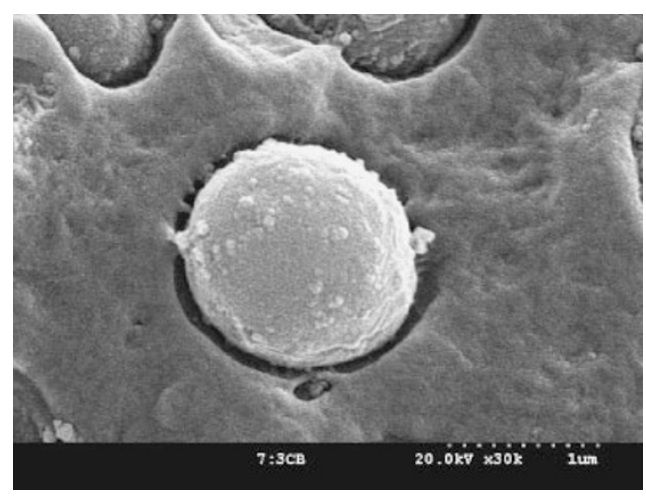

(a)

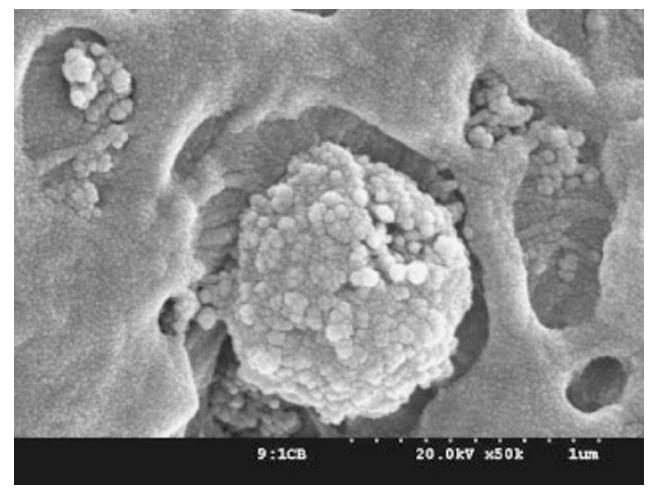

(b)

Figure 6. SEM micrographs of $\mathrm{CB}$ filled composites at high magnifications. (a) HDPE/sPS (70/30) and (b) HDPE/sPS (90/ $10)$.

close, which prepared at high speed (200 rpm).

The CB particles were preferentially attracted to the sPS phase. The sPS particle was coated with a rich CB containing layer, resulting in core-shell morphology. The sPS particles cannot be fully penetrated by CB, so $\mathrm{CB}$ is induced to segregate at the surface of sPS, as reported by Breuer et al. ${ }^{27}$ Though the surface tension of a conventional CB $(42.5 \mathrm{dyne} / \mathrm{cm})$ is much higher, the surface tension of sPS $(38 \mathrm{dyne} / \mathrm{cm})$ is higher than that of HDPE (31 dyne/cm). ${ }^{28}$ It is considered that the most of CB located in SPS domain and the rest of CB localized in HDPE matrix.

To confirm the location of CB, SEM micrographs magnified and the results are shown in Figure 6.

As depicted by SEM micrographs of the HDPE/ sPS/CB composites, Figure 6, CB appears at the surface of the spherical sPS particles. The lower content sPS composite (HDPE/sPS $=90 / 10$ ) was coated with a rich CB. CB is attracted to the sPS phase rather than to the HDPE.

In a high sPS content composite (for example 50 and $30 \mathrm{wt} \% \mathrm{sPS}$ ), the dispersion of sPS particles are homogeneous and the distance of sPS particles are very close. The CB-coated sPS particles could be 


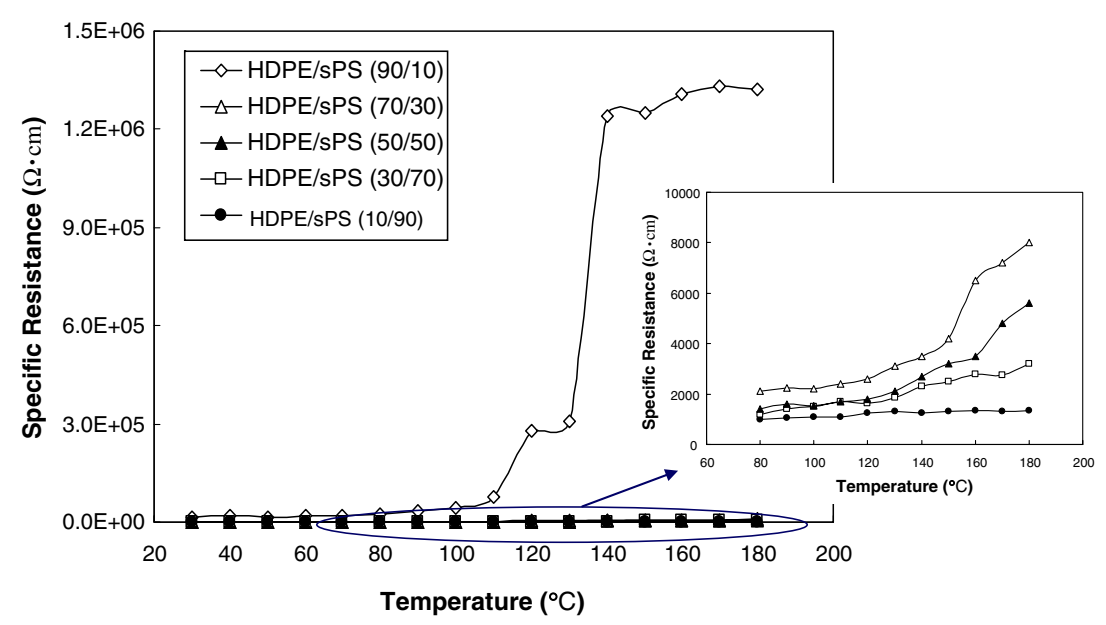

Figure 7. Effect of HDPE/sPS ratios on specific resistance of composites containing $10 \mathrm{phr}$ CB which prepared at $60 \mathrm{rpm}$.

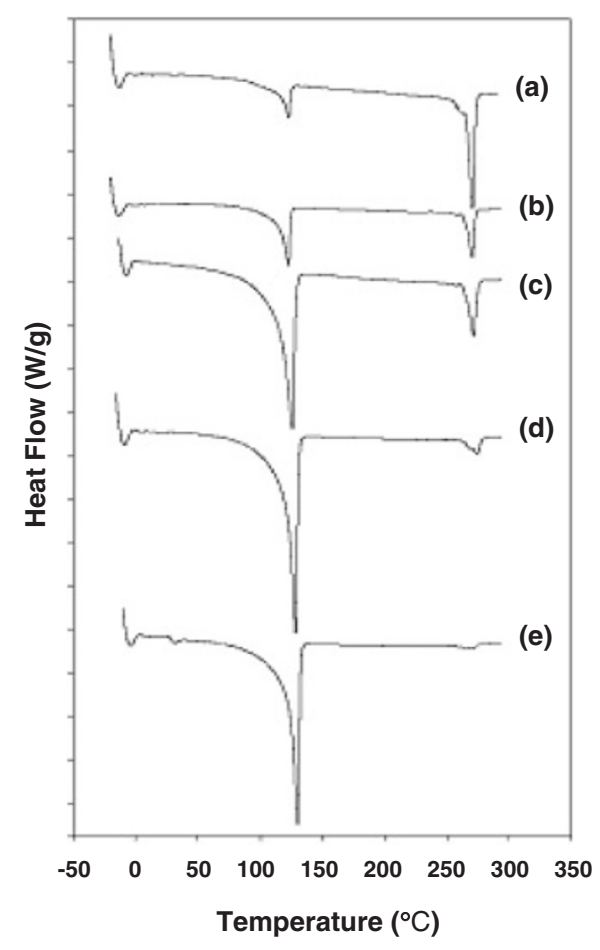

Figure 8. DSC thermograms on matrix content ratios (HDPE/ sPS, (a) 10/90, (b) 30/70, (c) 50/50, (d) 70/30, (e) 90/10) Carbon black content: $10 \mathrm{phr}$, Processing rotation speed: $60 \mathrm{rpm}$.

formed a continuous conductive network; therefore the PTC effect became weaker and weaker in these systems as depicted in Figure 7.

Figure 7 displayed the thermoelectric behaviors of these systems with a function of temperature.

The PTC effect could not be observed in high sPS content composite $(90,70,50,30 \mathrm{wt} \%$ sPS). The PTC effect at the melting point of the HDPE is observed and the value of PTC intensity was above 6 in low sPS content (10 wt \% sPS) composite. It is possible that in this system, the increase in the CBcoated sPS particle distance caused the increase in the resistivity. The significant NTC behaviors could

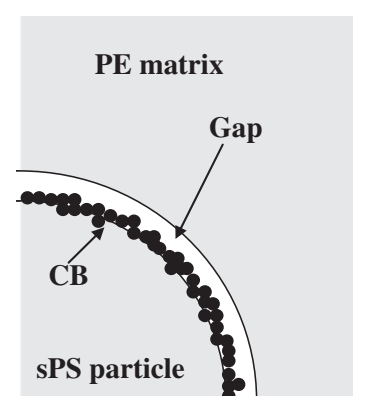

Figure 9. A schematic model of interface in HDPE/sPS/CB composite.

not be observed. The penetration of CB particles into the HDPE matrix could hardly observed in Figures 4 and 6 , preventing the formation of new conductive networks; hence the NTC effect could not observed.

As the results of morphological and thermoelectric characteristics, the HDPE/sPS/10-phr CB composites formed a continuous conductive network at high content of sPS.

To reveal the PTC mechanism of the composites, a DSC was used to study the melting behaviors of the HDPE/sPS composites with various sPS contents and the results are shown in Figure 8.

The melting of the HDPE and sPS crystallites was clearly observed which means two components are immiscible blends. The melting region of sPS was wider and those of HDPE were enlarged with increasing HDPE contents, while the melting peaks of HDPE and SPS were constants.

The schematic diagrams for the conducting mechanism of the HDPE/sPS/CB composites are shown in Figure 9. The similar conducting mechanism of the $\mathrm{PVDF} / \mathrm{UHMWPE} / \mathrm{CB}$ and PP/PMMA/CB composites has been reported in the literatures. ${ }^{29,30}$ When $\mathrm{CB}$ is added into an immiscible polymer blend, it is distributed in different proportions depending upon its affinity to the polymer components and the proc- 
essing history. There are two types of distribution that are beneficial to electrical conduction. One is distributed predominately in on continuous phase; the other case is located preferentially at the interface. ${ }^{31}$ When CB preferentially locates in one phase, the phase changes from a droplet domain into a continuous structure and the mixture of the two polymers becomes conductive. Therefore a resistivity increase due to the breaking of conductive paths on the sPS particles and a resistivity decrease due to the formation of conductive path in the HDPE matrix adjacent to the CB-coated sPS particle surface.

\section{CONCLUSION}

The temperature-conductivity characteristics of composites consisting of carbon black (CB)-filled high density polyethylene (HDPE)/syndiotactic polystyrene (sPS) were prepared by the conventional meltmixing procedure. In has been shown that the PTC effects of these composites are greatly affected by the sPS content, the mixing speed and morphology of the composites. Composites with a low sPS content and small-particle size sPS have a high room temperature resistivity value and a high PTC intensity. The morphology can influence the PTC effects of the composites. When $\mathrm{CB}$ is added into an immiscible polymer blend, it is distributed in different proportions depending upon its affinity to the polymer components. The CB particles were preferentially attracted to the sPS droplets which constitute the dispersed phase within the HDPE matrix. Thus, CB particles initially form conductive networks on the SPS phase; this is followed by distribution in the HDPE matrix, electrically connecting the CB-coated sPS particles. As low content of sPS and high processing speed, the composites exhibited high room temperature resistivity and high PTC intensity caused the CB-coated sPS particle distance increased. The high PTC intensity of CB-filled HDPE composites can be achieved by using a very high melting semicrystalline polymer as one of its component.

\section{REFERENCES}

1. M. Narkis, A. Ram, and F. Flashner, Polym. Eng. Sci., 18, 649 (1978).

2. C. Klason and J. Kubat, J. Appl. Polym. Sci., 19, 831 (1975).
3. J.-B. Donnet, Carbon, 32, 1305 (1994).

4. J. C. Huang, Adv. Polym. Technol., 14, 137 (1995).

5. M. L. Clingerman, E. H. Weber, J. A. King, and K. H. Shulz, Polym. Compos., 23, 911 (2002).

6. J. Meyer, Polym. Eng. Sci., 14, 706 (1974).

7. H. F. Xie, L. S. Dong, and J. Z. San, J. Appl. Polym. Sci., 95, 700 (2005).

8. M. Narkis, A. Ram, and Z. Stein, J. Appl. Polym. Sci., 25, 1515 (1980).

9. H. M. Al-Allak, A. W. Brinkman, and J. Woods, J. Mater. Sci., 28, 117 (1993).

10. H. Tang, J. H. Piao, X. F. Chen, Y. X. Lou, and S. H. Li, J. Appl. Polym. Sci., 48, 1795 (1993).

11. C.-M. Chan, Polym. Eng. Sci., 36, 495 (1996).

12. P. Mather and K. J. Thomas, J. Mater. Sci., 32, 1711 (1997).

13. K. Levon, A. Margolina, and A. Z. Patashinsky, Macromolecules, 26, 1261 (1993).

14. G. Geuskens, J. L. Gielens, D. Geshef, and R. Deltour, Eur. Polym. J., 23, 993 (1987).

15. G. Geuskens, E. D. Kezel, S. Blachers, and S. Brouers, Eur. Polym. J., 27, 1261 (1991).

16. C.-M. Chan, C.-L. Cheng, and M. Yuen, Polym. Eng. Sci., 37, 1127 (1997).

17. M. Narkis and A. J. Vaxman, J. Appl. Polym. Sci., 29, 1639 (1984).

18. J. Feng and C.-M. Chan, Polymer, 41, 4559 (2000).

19. I. Mironi-Harpaz and M. Narkis, J. Appl. Polym. Sci., 81, 104 (2001).

20. F. Kohler, U. S. Patent 3243 753, March 29, 1966.

21. Y. P. Mamunya, Y. Muzychenko, P. Possis, E. V. Lebedev, and M. I. Shut, Polym. Eng. Sci., 42, 90 (2002).

22. K. Ohe and Y. Naito, Jpn. J. Appl. Phys., 10, 99 (1971).

23. H. Xie, P. Deng, L. Dong, and J. Sun, J. Appl. Polym. Sci., 85, 2742 (2002).

24. J. Feng and C.-M. Chan, Polym. Eng. Sci., 39, 1207 (1999).

25. J. Feng and C.-M. Chan, Polym. Eng. Sci., 38, 1649 (1998).

26. F. Gubbels, R. Jerome, E. Vanlathem, R. Deltour, S. Blacher, and F. Brouers, Chem. Mater., 10, 1227 (1998).

27. S. Breuer, R. Tchoudakov, M. Narkis, and A. Siegmann, Polym. Eng. Sci., 40, 1015 (2000).

28. F. Gubbels, S. Blacher, E. Vanlathem, R. Jérome, R. Deltour, F. Brouers, and P. Teyssié, Macromolecules, 28, 1559 (1995).

29. I. Mironi-Harpaz and M. Narkis, Polym. Eng. Sci., 41, 205 (2001).

30. J. Feng, C. M. Chan, and J. X. Li, Polym. Eng. Sci., 43, 1058 (2003).

31. F. Gubbels, R. Jérome, P. Teyssié, E. Vanlathem, R. Deltour, A. Calderone, V. Parenté, and J. Brédas, Macromolecules, 27, 1972 (1994). 